\title{
Chronic fluoxetine treatment attenuates post-septic affective changes in the mouse
}

\author{
Sean T. Anderson ${ }^{\mathrm{a}}$, Sean Commins ${ }^{\mathrm{a}}$, Paul Moynagh ${ }^{\mathrm{b}}$, Andrew N. Coogan ${ }^{\mathrm{a}, *}$ \\ a Maynooth University Department of Psychology, National University of Ireland, Maynooth, Ireland \\ ${ }^{\mathrm{b}}$ Maynooth University Department of Biology, National University of Ireland, Maynooth, Ireland
}

\section{H I G H L I G H T S}

- LPS-induced sepsis leads to long-lasting changes in affective behaviours.

- Chronic fluoxetine treatment following recovery from sepsis attenuates such changes in affective behaviours.

- Chronic fluoxetine treatment also attenuates post-septic decreases in cell proliferation in the dentate gyrus, decreased expression of EGR1 in the CA1 and increased microglial activation in the hippocampus.

\section{A R T I C L E I N F O}

\section{Article history:}

Received 17 July 2015

Received in revised form 2 October 2015

Accepted 3 October 2015

Available online 9 October 2015

\section{Keywords:}

Sepsis

Fluoxetine

Neuoinflammation

EGR1

Depression

BrdU

\begin{abstract}
A B S T R A C T
It has been previously demonstrated that the induction of sepsis in rodents results in persistent impairments in affective and cognitive domains. In this study we have examined the impact of chronic treatment with the antidepressant fluoxetine on affective behaviours and hippocampal neuroinflammation and stem cell proliferation in animals that have previously undergone sepsis induced by peripheral treatment with lipopolysaccharide. We find that fluoxetine significantly attenuates post-septic increases in behavioural despair and motivated exploration, whilst also reversing the effects of previous sepsis on activated microglia and stem cell proliferation. These results indicate that conventional antidepressants may be effective in the management of mood disorders in survivors of sepsis.
\end{abstract}

(c) 2015 Elsevier B.V. All rights reserved.
It is increasingly recognised that survivors of sepsis may experience a long-lasting syndrome of cognitive and behavioural changes of uncertain aetiology that can significantly impact on quality of life [1]. Similar changes in cognitive and behavioural domains can be described in animal models of sepsis-survival, indicating that such approaches may be useful in understanding mechanisms and factors that shape post-septic impairments [2,3]. We have recently described that in a mouse model in which sepsis is induced by a sub-lethal treatment with lipopolysaccharide (LPS) there are long-lasting changes in affective domains, including increased behavioural despair and increases in anxiety-like behaviours [4]. In post-septic animals there is also decreased cellular proliferation in the dentate gyrus, altered hippocampal expression of the

* Corresponding author at: Maynooth University Department of Psychology, National University of Ireland, Maynooth, County Kildare, Ireland. Fax: +353 17084767 .

E-mail address: andrew.coogan@nuim.ie (A.N. Coogan). neural plasticity-related immediate early genes EGR1 and ARC, and evidence of increased and long-lasting neuroinflammation [4].

There are emerging links between neuroinflammation and affective disorders such as major depression that suggest that neuroimmune factors may be therapeutic targets in the treatment of mood disorders $[5,6]$. The selective serotonin reuptake inhibitor fluoxetine, a commonly used anti-depressant, has been described to exert anti-neuroinflammatory properties [7-9], as well as exerting effects on neurogenesis and neural plasticity [10,11]. Given the need to identify potential therapeutic strategies for the alleviation of post-septic symptoms, in the current study we examined whether chronic fluoxetine treatment following recovery from LPSinduced sepsis could attenuate some of the previously described affective and neurochemical changes observed in the mouse.

For the purpose of all experiments male C57BL/6 mice (Charles River, Kent, UK; $N=41$ ) were used. Animals were aged 8 weeks at the start of the experimental procedures. Animals were group housed in a 12:12 light:dark cycle for 2 weeks prior to LPS 


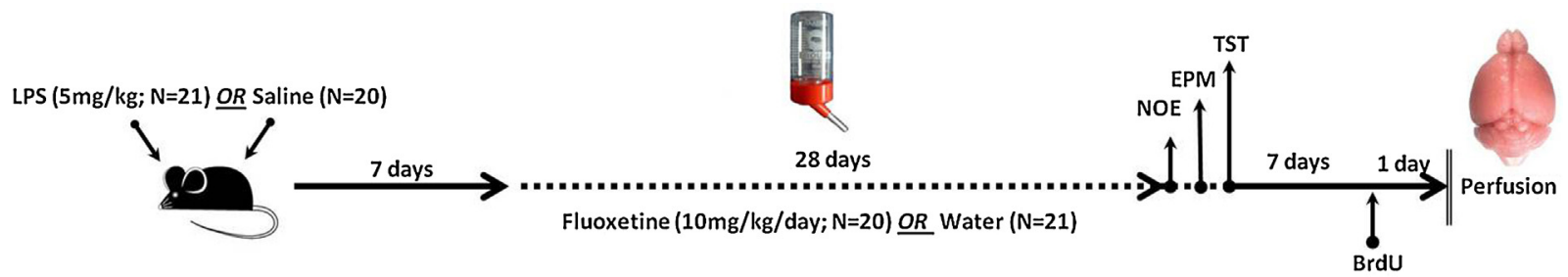

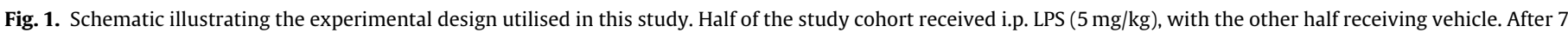

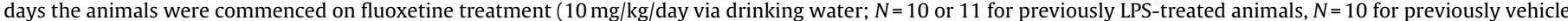

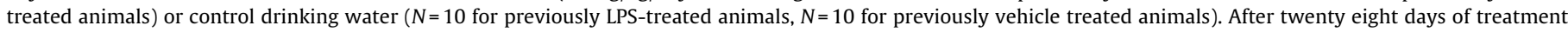

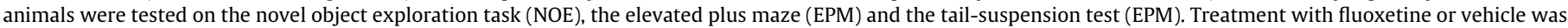
then discontinued for 7 days. All animals then received an i.p. treatment of BrdU $(50 \mathrm{mg} / \mathrm{kg})$ and were perfused $24 \mathrm{~h}$ later.

administration. Food and water were available ad libitum and temperature was $21 \pm 1{ }^{\circ} \mathrm{C}$ and humidity was $50 \pm 10 \%$. Animals remained housed in groups of 2-4 in polypropylene cages $(33 \mathrm{~cm}$ long $\times 15 \mathrm{~cm}$ wide $\times 13 \mathrm{~cm}$ high) with wood chip bedding and environmental enrichment (shredded paper and cardboard tubes). All procedures were approved by the Research Ethics Committee, National University of Ireland Maynooth, and were licensed by the Department of Health and Children, Ireland under statutory instrument No. 543 of 2012 and the European directive 2010/63/EU.

The experimental regime employed is represented in Fig. 1. For the induction of sepsis, animals were given a single i.p. treatment of LPS ( $5 \mathrm{mg} / \mathrm{kg}$; serotype 0111.B4, Sigma Ireland) or control animals were treated with vehicle sterile saline. One week after LPS/vehicle treatment, animals were treated with fluoxetine $(10 \mathrm{mg} / \mathrm{kg}$, Tocris Bioscience, UK) in standard drinking water, or just water for control. Fresh fluoxetine was given every second day; animals and water were weighed to ensure the correct dosage was maintained. Animals were given fluoxetine or vehicle for 28 days before behavioural testing began and throughout the duration of behavioural testing. Animals were tested on the novel object exploration task followed by the elevated plus maze and then the tail suspension test. All behavioural tests were carried out as previously described, and the selection of behavioural tests was made on the basis of results from our previous study regarding post-septic affective and cognitive changes in the mouse [4]. Briefly, for the tail suspension test mice were attached to a support raised to a height of $121 \mathrm{~cm}$ using tape placed $1 \mathrm{~cm}$ from the tip of their tales for six minutes. Mice were suspended for six minutes each, with immobility (complete absence of movement) being recorded throughout the entire six minutes. For the novel object exploration task animals were first habituated to an arena of $50 \mathrm{~cm}$ and then underwent 5 trials lasting two minutes being habituated to two objects. On the fifth trial, one familiar object was replaced with a novel object. Animals were measured on their time touching, and number of nose touches for each object. As such we primarily use this task as a measure of exploratory motivation, but also calculate preference ratios for exploration of the novel object in the probe trial as a measure of working memory. For the elevated plus maze, the maze consisted of a centre area of diameter $13.5 \mathrm{~cm}$ at a height of $20 \mathrm{~cm}$, from which four arms extended of length $34.5 \mathrm{~cm}$, width $5 \mathrm{~cm}$. Two arms were open without walls, while the other two were enclosed by high walls. Entrance to an arm was counted where all four of an animals' paws were within the arm. Animals underwent one five-minute testing session each.

To assess the rate of cellular proliferation in the hippocampus mice were injected i.p. with $50 \mathrm{mg} / \mathrm{kg}$ BrdU one week after the conclusion of behavioural testing. $24 \mathrm{~h}$ later mice were terminally anaesthetised with $0.1 \mathrm{ml}$ sodium pentobarbital (Euthatal, Merial Animal Health, UK) and perfused transcardially with $0.9 \%$ saline, with brains fixed in $4 \%$ paraformaldehyde (Sigma Ireland) in $0.1 \mathrm{M}$ phosphate buffer (PB), pH 7.4 at $4{ }^{\circ} \mathrm{C}$. Brains were then cryoprotected in $0.1 \mathrm{M}$ PB containing 30\% sucrose, frozen and sectioned coronally at $30 \mu \mathrm{m}$. Immunohistochemistry for IBA1 (1:3000, rabbit polyclonal primary antibody, Wako, Denmark; 01919741), EGR1 (1:3000, rabbit polyclonal primary antibody, Santa Cruz Biotechnology; sc-189) and BrdU (1:200, rat monoclonal primary antibody, AbD Serotec, Oxford, UK; MCA2060) was carried out following a standard Avidin-Biotin Complex/Nickel DAB protocol as previously described $[4,12]$. Photomicrographs of sections were taken using a digital camera connected to an Olympus BX-51 light microscope under constant light intensity. Morphology and number of active microglia (stages 3-5 of Kreutzberg [13]) stained with IBA1 were assessed under $100 \times$ magnification within a pre-defined area. EGR1 immunoreactivity was assessed by optical density with ImageJ 1.43 (NIH, USA). BrdU immunopositive cells in the dentate gyrus were counted by eye. All image assessment was carried out by an experimenter blind to the experimental manipulation.

Data from the behavioural experiments and neurochemical data were assessed via a factorial multivariate analysis of variance (MANOVA). The dependent variables for the behavioural data were time spent immobile on the tail suspension test, time spent in open arms on the elevated plus maze and touches to both objects on the probe trial of the novel object exploration task. The factors were prior LPS treatment or vehicle and fluoxetine treatment or vehicle. For immunohistochemical data the dependent variables were cells incorporating BrdU, optical density for EGR1 and the number of IBA1-positive microglia with activated-like morphology. For post-hoc analysis Bonferroni corrections were applied. $P<0.05$ was considered statistically significant. All data are presented as means and standard error of the mean.

As previous data from our laboratory had demonstrated that LPS-induced sepsis was associated with a persistent increase in immobility in the tail suspension test, decreased object exploration in the probe trial of a novel object exploration task and decreased time spent in the open arms of the elevated plus maze, we examined whether these post-sepsis-associated changes would be ameliorated by chronic fluoxetine treatment (Fig. 2). MANOVA results from the behavioural experiments indicate that there is a statistically significant interaction between LPS treatment and subsequent fluoxetine treatment, and that the magnitude of such an effect is large $\left(F_{3,37}=20.43, P<0.001\right.$, partial eta squared $\left.=0.629\right)$. There were also significant main effects of both LPS treatment $\left(F_{3,37}=22.17, P<0.001\right.$, partial eta squared $\left.=0.65\right)$ and fluoxetine treatment $\left(F_{3,37}=5.5, P<0.01\right.$, partial eta squared $\left.=0.32\right)$. Considering the outcomes of the individual behavioural tests, there were statistically significant interactions between LPS treatment and fluoxetine treatment for the tail suspension test $\left(F_{1,37}=36.1, P<0.001\right.$, partial eta squared $=0.49$ ) and the novel object exploration test $\left(F_{1,37}=5.27, P<0.05\right.$, partial eta squared $\left.=0.12\right)$, but not the elevated plus maze $\left(F_{1,37}=1.88, P=0.163\right)$. For the novel object exploration test, there were no significant effects on the preference for exploration of the novel object versus the familiar object, as has been 
A TailSuspension Test

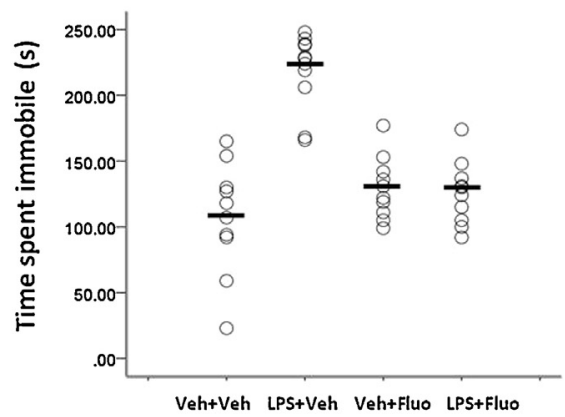

B Elevated Plus Maze

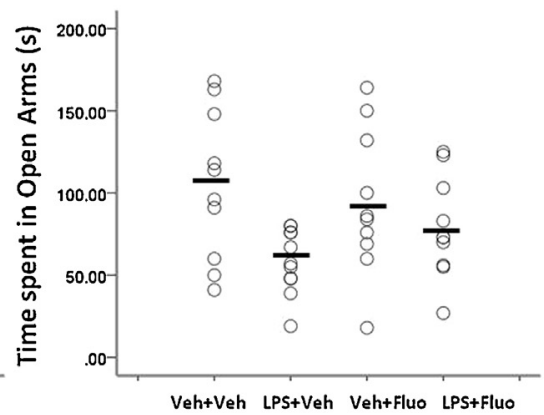

C Novel Object Exploration

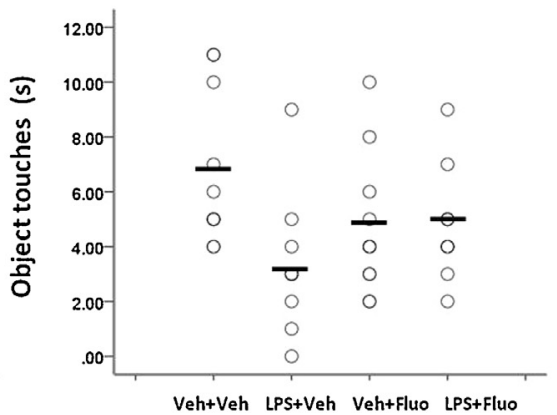

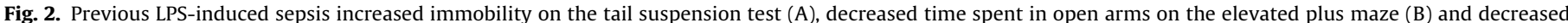

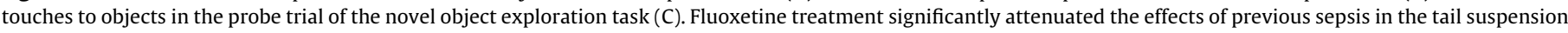

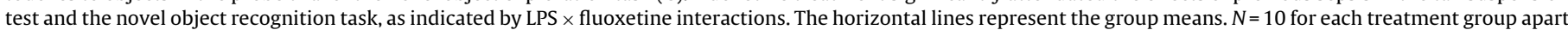
from LPS + Veh group where $N=11$.

previously reported [4]. These results indicate that chronic fluoxetine treatment after the induction of sepsis significantly attenuates behavioural changes observed on the tail suspension test and the novel object exploration task.

Analysis of the immunohistochemical data likewise indicates that post-septic treatment with fluoxetine attenuates some effects previously described in post-septic animals [4]. Considering BrdU incorporation in the dentate gyrus, EGR1 expression in the CA1 and hippocampal expression of IBA1 (Fig. 3), MANOVA demonstrates a significant, large magnitude interaction between LPS treatment and fluoxetine treatment $\left(F_{3,37}=27.1, P<0.001\right.$, partial eta squared $=0.699$ ). There were also significant main effects of both LPS treatment $\left(F_{3,37}=37.9, P<0.001\right.$, partial eta squared $=0.77)$ and fluoxetine treatment $\left(F_{3,37}=33.74, P<0.001\right.$, partial eta squared $=0.75$ ). Analysis of the separate dependent variables shows significant $\mathrm{LPS} \times$ fluoxetine interactions for $\mathrm{BrdU}$ $\left(F_{1,37}=23.8, P<0.001\right.$, partial eta squared $\left.=0.391\right)$, EGR1 $\left(F_{1,37}=6.3\right.$, $P<0.05$, partial eta squared $=0.146)$ and $\operatorname{IBA} 1\left(F_{1,37}=42.9, P<0.001\right.$, partial eta squared $=0.537$ ).
These results indicate that in murine survivors of LPS-induced sepsis that chronic fluoxetine treatment can attenuate both affective behavioural and hippocampal neurochemical changes. Of particular interest is that fluoxetine treatment was not administered until 7 days after LPS treatment, and as such did not impact on post-septic effects by modulating sepsis severity. Previous studies have shown that in animal models strategies that target the acute phase of sepsis lead to diminution of post-septic cognitive and behavioural effects [4,14-16]. However, for the management of post-septic impairments in patients it will be important to identify strategies that may be effective in survivors, aside from the obvious need to identify therapeutics that reduce sepsis severity and increase survival. From this point of view, it is encouraging that a commonly prescribed antidepressant, fluoxetine, appears to be effective in this pre-clinical study at ameliorating postseptic effects in the mouse. Interestingly, a previous study reported that acute treatment with the antidepressant imipramine reversed depressive-symptoms in rats 10 days following caecal ligation and perforation-induced sepsis [17], a finding which is similar to that
A $\operatorname{BrdU}$
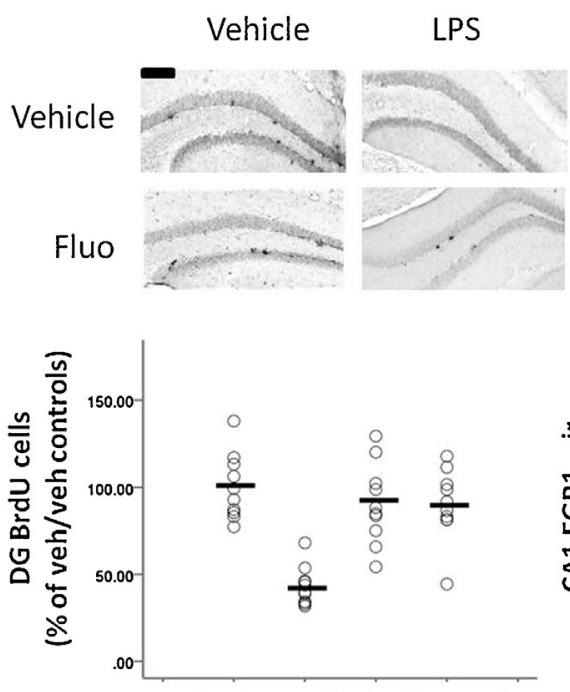

Veh+Veh LPS+Veh Veh+Fluo LPS+Fluo
B $E G R 1$

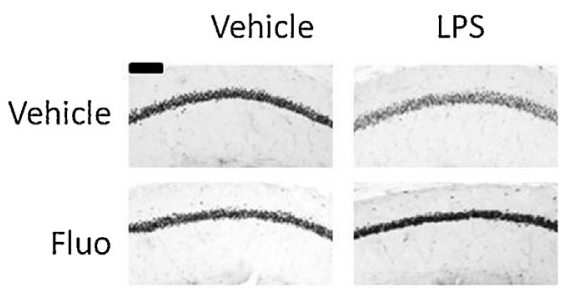

$\underline{C} \mid B A-1$
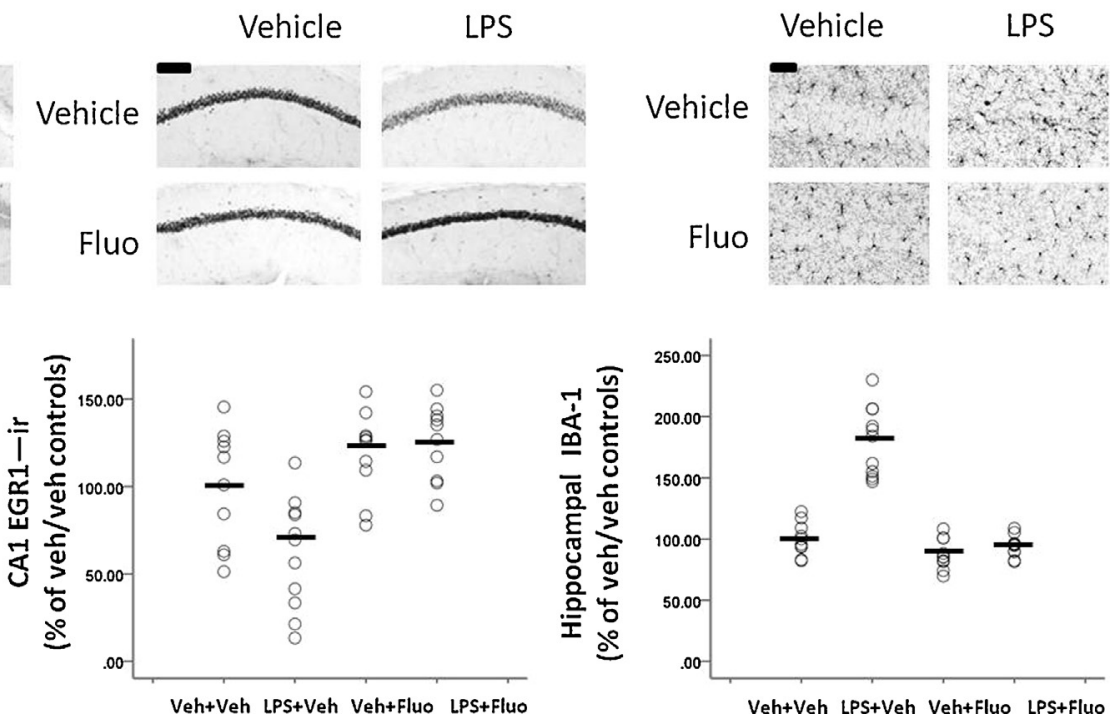
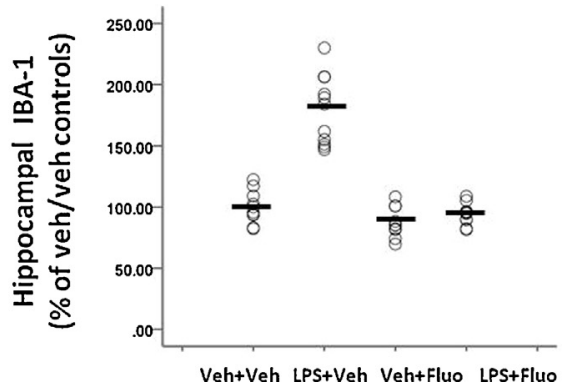

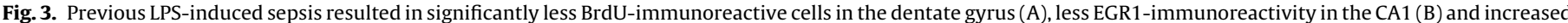

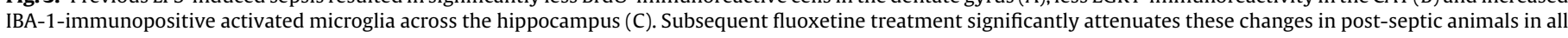
three antigens, as indicated by LPS $\times$ fluoxetine interactions. Scale bar in the photomicrographs $=100 \mu \mathrm{m}$. $N=10$ or $N=11$ for each group. 
reported for acute treatment with fluoxetine in LPS-induced sepsis survivor mice [4]. However, no previous study has addressed the efficacy of post-septic chronic anti-depressant treatment, a treatment modality that is likely to be of greater translatable significance and predictive validity than acute treatments. Further, no previous study has examined the impact of antidepressant treatment on neurochemical processes that may underpin affective changes in sepsis-survivor animals. One previous study indicated that postseptic chronic treatment with anti-high mobility group box 1 antibodies also attenuated cognitive impairment and hippocampal dendritic spine loss [18], indicating that there may be numerous mechanisms through which post-septic changes in behaviour and cognition may be targeted.

The aetiology of post-septic encephalopathy is likely to be complex, involving factors such as impaired blood-brain barrier function, oxidative stress, neuroinflammation, decreased neural plasticity and altered EEG rhythms [19]. These mechanisms are likely to be highly interconnected; for example, sepsis-induced activation of microglia may underlie concomitant synaptic dysfunction and increased oxidative damage [20,21]. It is interesting to note that in the present study that the fluoxetine-induced attenuation of affective behaviours was found in parallel with decreased microglial activation and rescue of cellular proliferation in the dentate gyrus and EGR1 levels in the CA1. Previous studies have indicated an anti-neuroinflammatory role for fluoxetine via upregulation of anti-inflammatory mediators such as interleukin 4 [9] and an effect in downregulating oxidative stress [22]. Given that microglia may be important regulators of neurogenesis in models of affective disorders [23], there may be a direct link between microglial activation and altered rates of hippocampal stem cell proliferation following sepsis that is acted on by fluoxetine. Interestingly fluoxetine has been shown to be effective in restoring rates of neural stem cell proliferation in a model of depression involving pre-natal LPS treatment [24].

There are a number of important caveats and limitations of the current study: we did not assess profiles of pro- and anti-inflammatory cytokines and important mediators of neuroinflammation, and as such we lack mechanistic insight into how fluoxetine may exert its seemingly beneficial actions in post-septic mice; further, we did not examine factors known to regulate neurogeneis, such as brain-derived neurotrophic factor. Future studies should address these questions. The results of the current study also raise a number of further questions: would other anti-depressants be effective in ameliorating post-septic affective changes; would fluoxetine be effective in the treatment of sepsis survivor patients who display mood disorders (and/or cognitive impairments); is there a time-window in which post-septic treatment may only be effective; is there utility in the combination of fluoxetine (or other antidepressants) with drugs such as cecoxilib or minocycline that directly target neuroinflammation? There is a clear need for further pre-clinical and clinical studies to guide the future treatment of survivors of sepsis.

\section{Acknowledgements}

We acknowledge funding from John and Pat Hume Scholarship award from Maynooth University to support S.T.A.

\section{References}

[1] C.N. Widmann, M.T. Heneka, Long-term cerebral consequences of sepsis, Lancet Neurol. 13 (6) (2014) 630-636.
[2] M. Weberpals, M. Hermes, S. Hermann, M.P. Kummer, D. Terwel, A. Semmler, M. Berger, M. Schäfers, M.T. Heneka, NOS2 gene deficiency protects from sepsis-induced long-term cognitive deficits, J. Neurosci. 29 (45) (2009) 14177-14184

[3] P. Bossù, D. Cutuli, I. Palladino, P. Caporali, F. Angelucci, D. Laricchiuta, F. Gelfo, P. De Bartolo, C. Caltagirone, L. Petrosini, A single intraperitoneal injection of endotoxin in rats induces long-lasting modifications in behavior and brain protein levels of TNF- $\alpha$ and IL-18, J. Neuroinflammation 9 (2012) 101.

[4] S.T. Anderson, S. Commins, P.N. Moynagh, A.N. Coogan, Lipopolysaccharide-induced sepsis induces long-lasting affective changes in the mouse, Brain Behav. Immun. 43 (2015) 98-109.

[5] A. Cattaneo, F. Macchi, G. Plazzotta, B. Veronica, L. Bocchio-Chiavetto, M.A. Riva, C.M. Pariante, Inflammation and neuronal plasticity: a link between childhood trauma and depression pathogenesis, Front. Cell. Neurosci. 9 (2015) 40.

[6] E. Setiawan, A.A. Wilson, R. Mizrahi, P.M. Rusjan, L. Miler, G. Rajkowska, I. Suridjan, J.L. Kennedy, P.V. Rekkas, S. Houle, J.H. Meyer, Role of translocator protein density, a marker of neuroinflammation, in the brain during major depressive episodes, JAMA Psychiatry 72 (3) (2015) 268-275.

[7] D. Liu, Z. Wang, S. Liu, F. Wang, S. Zhao, A. Hao, Anti-inflammatory effects of fluoxetine in lipopolysaccharide (LPS)-stimulated microglial cells, Neuropharmacology 61 (4) (2011) 592-599.

[8] C. Roumestan, A. Michel, F. Bichon, K. Portet, M. Detoc, C. Henriquet, D. Jaffuel, M. Mathieu, Anti-inflammatory properties of desipramine and fluoxetine, Respir. Res. 8 (2007) 35.

[9] S. Alboni, C. Benatti, C. Montanari, F. Tascedda, N. Brunello, Chronic antidepressant treatments resulted in altered expression of genes involved in inflammation in the rat hypothalamus, Eur. J. Pharmacol. 721 (December 5 $(1-3))(2013) 158-167$

[10] O.F. O'Leary, J.F. Cryan, A ventral view on antidepressant action: roles for adult hippocampal neurogenesis along the dorsoventral axis, Trends Pharmacol. Sci. 35 (12) (2014) 675-687.

[11] E. Castrén, T. Rantamäki, The role of BDNF and its receptors in depression and antidepressant drug action: reactivation of developmental plasticity, Dev. Neurobiol. 70 (5) (2010) 289-297.

[12] S.T. Anderson, E.K. O'Callaghan, S. Commins, A.N. Coogan. Does prior sepsis alter subsequent circadian and sickness behaviour response to lipopolysaccharide treatment in mice? J. Neural Transm.

[13] G.W. Kreutzberg, Microglia: a sensor for pathological events in the CNS, Trends Neurosci. 19 (8) (1996) 312-318.

[14] R. Gao, Y.H. Tang, J.H. Tong, J.J. Yang, M.H. Ji, S.H. Zhu, Systemic lipopolysaccharide administration-induced cognitive impairments are reversed by erythropoietin treatment in mice, Inflammation 38 (5) (2015) 1949-1958.

[15] H. Wei, X. Cao, Q. Zeng, F. Zhang, Q. Xue, Y. Luo, J.W. Lee, B. Yu, X. Feng, Ghrelin inhibits proinflammatory responses and prevents cognitive impairment in septic rats, Crit. Care Med. 43 (May (5)) (2015) e143-e150.

[16] M. Michels, L.G. Danieslki, A. Vieira, D. Florentino, D. Dall'igna, L. Galant, B. Sonai, F. Vuolo, F. Mina, B. Pescador, D. Dominguini, T. Barichello, J. Quevedo, F. Dal-Pizzol, F. Petronilho, CD40-CD40 ligand pathway is a major component of acute neuroinflammation and contributes to long-term cognitive dysfunction after sepsis, Mol. Med. 21 (March 26) (2015) 219-226.

[17] L. Tuon, C.M. Comim, M.M. Atunes, L.S. Constantino, R.A. Machado, I. Izquierdo, J. Quevedo, F. Dal-Pizzol, Imipramine reverses the depressive symptoms in sepsis survivor rats, Intensive Care Med. 33 (2007) 2165-2167.

[18] S.S. Chavan, P.T. Huerta, S. Robbiati, S.I. Valdes-Ferrer, M. Ochani, M. Dancho, M. Frankfurt, B.T. Volpe, K.J. Tracey, B. Diamond, HMGB1 mediates cognitive impairment in sepsis survivors, Mol. Med. 18 (2012) 930-937.

[19] D. Annane, T. Sharshar, Cognitive decline after sepsis, Lancet Respir. Med. 3 (1) (2015) 61-69.

[20] C.A. Moraes, G. Santos, T.C. Spohr, J.C. D’Avila, F.R. Lima, C.F. Benjamim, F.A. Bozza, F.C. Gomes, Activated microglia-induced deficits in excitatory synapses through IL-1 $\beta$ : implications for cognitive impairment in sepsis, Mol. Neurobiol. 52 (1) (2015) 653-663.

[21] M. Michels, A.S. Vieira, F. Vuolo, H.G. Zapelini, B. Mendonça, F. Mina, D Dominguini, A. Steckert, P.F. Schuck, J. Quevedo, F. Petronilho, F. Dal-Pizzol, The role of microglia activation in the development of sepsis-induced long-term cognitive impairment, Brain Behav. Immun. 43 (2015) 54-59.

[22] E.S. Chung, Y.C. Chung, E. Bok, H.H. Baik, E.S. Park, J.Y. Park, S.H. Yoon, B.K. Jin, Fluoxetine prevents LPS-induced degeneration of nigral dopaminergic neurons by inhibiting microglia-mediated oxidative stress, Brain Res. 1363 (2010) 143-150.

[23] T. Kreisel, M.G. Frank, T. Licht, R. Reshef, O. Ben-Menachem-Zidon, M.V. Baratta, S.F. Maier, R. Yirmiya, Dynamic microglial alterations underlie stress-induced depressive-like behavior and suppressed neurogenesis, Mol. Psychiatry 19 (6) (2014) 699-709.

[24] Y.L. Lin, S. Wang, Prenatal lipopolysaccharide exposure increases depression-like behaviors and reduces hippocampal neurogenesis in adult rats, Behav. Brain Res. 259 (2014) 24-34. 